\title{
A Radiative Transfer Framework for Spatially-Correlated Materials
}

\author{
ADRIAN JARABO, Universidad de Zaragoza - I3A \\ CARLOS ALIAGA, Universidad de Zaragoza - I3A, and Desilico Labs \\ DIEGO GUTIERREZ, Universidad de Zaragoza - I3A
}
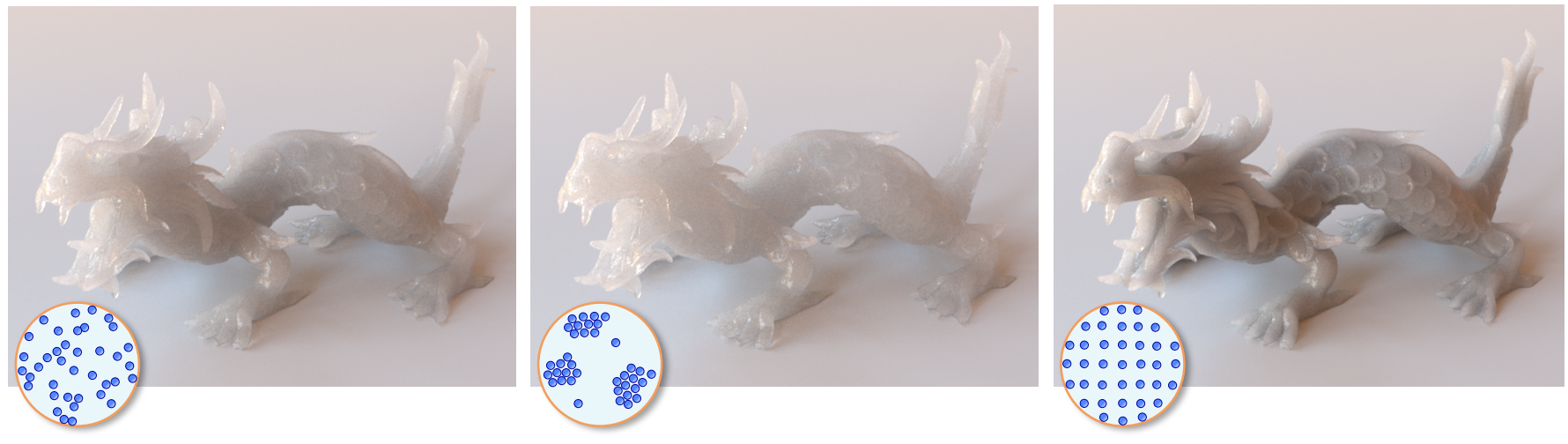

Fig. 1. Spatial correlation in media leads to non-exponential light transport, which significantly affects appearance. The image shows volumetric renderings of translucent dragons made of materials with the same density per unit differential volume $\bar{\mu}=10$ (isotropic, albedo $\Lambda=.8$ ), but different correlations. Left: using classic light transport, where material particles are assumed to be uncorrelated. Middle and right: positive and negative correlation, respectively, using our novel framework for spatially-correlated materials. The insets show illustrative views of scatterer correlation for each dragon.

We introduce a non-exponential radiative framework that takes into account the local spatial correlation of scattering particles in a medium. Most previous works in graphics have ignored this, assuming uncorrelated media with a uniform, random local distribution of particles. However, positive and negative correlation lead to slower- and faster-than-exponential attenuation respectively, which cannot be predicted by the Beer-Lambert law. As our results show, this has a major effect on extinction, and thus appearance. From recent advances in neutron transport, we first introduce our Extended Generalized Boltzmann Equation, and develop a general framework for light transport in correlated media. We lift the limitations of the original formulation, including an analysis of the boundary conditions, and present a model suitable for computer graphics, based on optical properties of the media and statistical distributions of scatterers. In addition, we present an analytic expression for transmittance in the case of positive correlation, and show how to incorporate it efficiently into a Monte Carlo renderer. We show results with a wide range of both positive and negative correlation, and demonstrate the differences compared to classic light transport.

CCS Concepts: • Computing methodologies $\rightarrow$ Rendering; Reflectance modeling;

Authors' addresses: Adrian Jarabo, Universidad de Zaragoza - I3A, ajarabo@unizar.es; Carlos Aliaga, Universidad de Zaragoza - I3A, and Desilico Labs, carlos.aliaga@desilico. tech; Diego Gutierrez, Universidad de Zaragoza - I3A, diegog@unizar.es.

Permission to make digital or hard copies of all or part of this work for personal or classroom use is granted without fee provided that copies are not made or distributed for profit or commercial advantage and that copies bear this notice and the full citation on the first page. Copyrights for components of this work owned by others than ACM must be honored. Abstracting with credit is permitted. To copy otherwise, or republish, to post on servers or to redistribute to lists, requires prior specific permission and/or a fee. Request permissions from permissions@acm.org.

(c) 2018 Association for Computing Machinery.

0730-0301/2018/8-ART83 \$15.00

https://doi.org/10.1145/3197517.3201282
Additional Key Words and Phrases: Spatially-Correlated Transport, NonExponential Light Transport, Correlated Radiative Transfer

\section{ACM Reference Format:}

Adrian Jarabo, Carlos Aliaga, and Diego Gutierrez. 2018. A Radiative Transfer Framework for Spatially-Correlated Materials. ACM Trans. Graph. 37, 4, Article 83 (August 2018), 13 pages. https://doi.org/10.1145/3197517.3201282

\section{INTRODUCTION}

Volumetric appearances are ubiquitous in the real world, from translucent organic materials to clouds, smoke, or densely packed granular media. Voxel-based representations with anistropic scattering functions [Heitz et al. 2015; Jakob et al. 2010] have been widely used in recent years to represent the appearance of complex geometries such as trees [Loubet and Neyret 2017; Neyret 1998], cloth and hair [Aliaga et al. 2017; Khungurn et al. 2015; Schröder et al. 2011; Zhao et al. 2011], or particles' aggregates [Meng et al. 2015; Moon et al. 2007; Müller et al. 2016].

Many translucent objects and participating media present a strong spatial correlation between scatterers ${ }^{1}$ [Coquard and Baillis 2006; Knyazikhin et al. 1998; Lovejoy et al. 1995], where scatterers' densities are non-uniform within a differential volume. The aerosol of clouds, for instance, tends to form clusters, resulting in areas with very different optical thicknesses [Marshak et al. 1998]. As a result, the probability of a photon interacting with a scatterer inside each differential volume is also non-uniform, which in turn has a great effect in the final appearance, as Figure 1 shows.

Most previous works in graphics have assumed an uncorrelated distribution of scatterers, considering only spatial correlation at a

\footnotetext{
${ }^{1}$ Following other works' terminology, through the paper we use the term "scatterers" for all particles in the media, including perfect absorbers.
} 

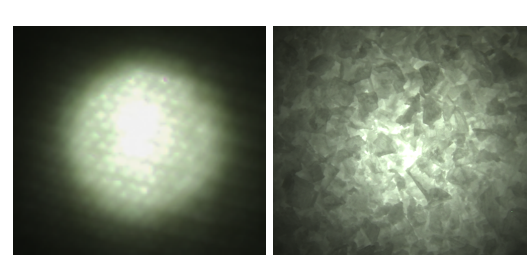

\begin{tabular}{lc} 
Media & $R^{2}$ \\
\hline Milk $^{*}$ & 0.99 \\
Black Fabric & 0.87 \\
White Fabric & 0.76 \\
Maldon Salt & 0.76 \\
Sugar & 0.69 \\
\hline
\end{tabular}

Fig. 2. Left: Photographs of spatially-correlated media (white fabric, and maldon salt), lit from behind using a mobile flash. Right: We evaluate the transmittance of different media, by fitting measurements at different optical thickness to the exponential decay predicted by the classic Beer-Lambert law. As expected, a diluted liquid such as milk (marked with an asterisk) shows a very close fit to the exponential decay (measured using the $R^{2}$ metric); however, transmittance in spatially-correlated media cannot be modeled using classic radiative transfer. Details on the experiment can be found in Section S.9 in the supplemental.

macroscopic scale as heterogeneous media. This results in the wellknown exponential transmittance predicted by the Beer-Lambert law. However, in the presence of correlation at differential-volume scale, the predictions of the Radiative Transfer Equation (RTE) [Chandrasekhar 1960] break, and therefore attenuation is no longer exponential (see Figure 2): In such cases, negatively-correlated media lead to faster-than-exponential transmittance, whereas positive correlation leads to slower-than-exponential transmittance [Davis et al. 1999]. Works rendering granular aggregates observed such nonexponential transmittance; however, they either formulate it in an uncorrelated radiative (exponential) framework [Meng et al. 2015], precalculate the full light transport explicitly [Moon et al. 2007], or combine both approaches [Müller et al. 2016].

In this work we introduce a theoretical framework for simulating light transport in spatially-correlated media, which accounts for the local structure of scatterers. Our framework builds upon the well-established radiative theory, and leverages recent advances in non-classical transport in the neutron transport field: We extend the Generalized Boltzmann Equation (GBE) [Larsen 2007], which generalizes the RTE to correlated media, and lift its main limitations, leading to a general framework suitable for computer graphics. In addition, we present an analytic expression of transmittance for positive correlation, leading to a compact representation of directionallydependent spatial correlation based on a gamma distribution of scatterers. We also present efficient sampling techniques, enabling the use of our model within any existing volumetric renderer. Our framework is able to accurately simulate light transport inside correlated media. We show results with a wide range of correlations, both negative and positive, and demonstrate the differences with classic (uncorrelated) light transport. Our model is general and intuitive, and can be seen as complementary in the spatial domain to angular anisotropy in media [Heitz et al. 2015; Jakob et al. 2010]. It might also be useful in other areas such as volumetric level of detail, or accelerating light transport using similarity theory.

Overview. The technical sections of the paper are organized as follows: We first present a general background of radiative transport in uncorrelated media, a brief summary of the effect of spatial correlation on extinction, and the Generalized Boltzmann Equation (Section 3). Unfortunately, the original formulation of the GBE presents some simplifying assumptions valid for neutron transport in reactors, but that limit its applicability in rendering. In Section 4 we present our Extended GBE, which lifts the limitations of the original GBE to support more general media, and include a thorough analysis of its boundary conditions. Finally, in Section 5 we propose an appearance model for positively-correlated media based on local optical parameters, which is intuitive and easy to manipulate, and which can be plugged directly into our Extended GBE.

\section{RELATED WORK}

Volumetric light transport. Simulating light transport in participating media has a long history in computer graphics (see e.g. [Gutierrez et al. 2008]). Existing methods aim to efficiently solve the RTE [Chandrasekhar 1960] by means of path tracing [Lafortune and Willems 1996; Veach 1997], photon mapping [Jensen 2001], photon beams [Jarosz et al. 2011], or a combination of these techniques [Křivánek et al. 2014]. Our framework is independent of the particular algorithm used for rendering. Jakob et al. [2010] extended the RTE to account for directional (angular) anisotropy. Later, Heitz et al. [2015] further extended this model with the SGGX microflakes distribution. While these works focus on the local angular dependence of scattering and extinction, they still assume that the scatterers are uncorrelated, distributed uniformly in the spatial domain. Our work is orthogonal to these approaches, focusing on the effects of spatial correlation.

Volumetric representation of appearance. Volumetric representations of explicit geometry have been successfully used to approximate complex appearances. Meng et al. [2015] used a classical radiative approximation of light transport in particulate media for efficient rendering. Fiber-level cloth appearance models, based either on micro-CT geometry [Zhao et al. 2011, 2012] or procedural modeling [Schröder et al. 2011], have used volumetric anisotropic representations for rendering high-detailed garments [Aliaga et al. 2017], similar in quality to explicit fiber representations [Khungurn et al. 2015]. Zhao et al. [2016] presented an optimization-based approach to downsample volumetric appearance representation by altering the rendering parameters (scattering and phase function) to match the desired appearance. All these works make again the assumption of perfect decorrelation of the scatterers in the medium. Our theoretical framework departs from this assumption.

Correlated volumetric media. Correlated volumetric media have been studied in computational transport in fields such as nuclear engineering [Camminady et al. 2017; Larsen and Vasques 2011; Levermore et al. 1986], atmospheric sciences [Davis and Marshak 2004; Davis et al. 1999; Newman et al. 1995], or thermal propagation [Bellet et al. 2009; Coquard and Baillis 2006; Taine et al. 2010], leading to non-classical transport theories [Frank and Goudon 2010; Larsen 2007]. Non-classical transport has been however largely unexplored in graphics: The first work modeling non-exponential flights in graphics is the work of Moon et al. [2007], which precomputed transport functions of granular materials as a set of homogeneous 

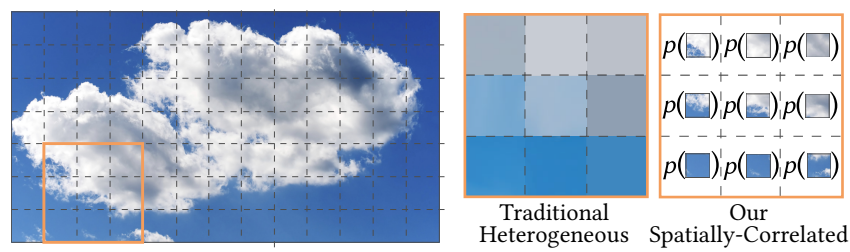

Fig. 3. Difference between heterogeneous and spatially-correlated media, from a computer graphics perspective. Heterogeneous media assume heterogeneity at macroscopic level, but local homogeneity at each differential volume $\mathrm{d} V(\mathbf{x})$ (see "Traditional Heterogeneous", represented as solid colors per voxel). Instead, our model for spatially-correlated media takes into account the uneven distribution of scatterers within each $\mathrm{d} V(\mathrm{x})$ (represented on the right as probabilities of extinction $p(\cdot))$.

shells. However, they required precomputing all light transport operators rather than attempting to express such non-exponential flights into a new radiative transport theory, and did not take into account the effect of correlation at boundaries. Müller et al. [2016] later used a similar approach in combination with other volumetric estimators for rendering heterogeneous discrete media. Concurrently to us, Wrenninge et al. [2017] used non-exponential flights for increased artist control on volumetric light transport, but omitted the underlying theory, and did not relate their model with the physical process of extinction. More formally, d'Eon analyzed rigorously the effect of isotropic non-Poissonian extinction on the diffusion (multiple scattering) regime [2014b;2016a], and discussed the connections between graphics and non-classical transport, including the limitations of such theories to be used in rendering [d'Eon 2014a, 2016b]. We generalize these works, offering a non-classic transport theory suitable for rendering, and introduce an intuitive formulation for rendering spatially-correlated media based on local optical parameters.

\section{RADIATIVE TRANSPORT IN CORRELATED MEDIA}

In this section, we first introduce light transport in participating media as modeled by the Radiative Transfer Equation (RTE) [Chandrasekhar 1960] (Section 3.1). We then describe the notion of spatial correlation in media, and its effect on light extinction (Section 3.2), as well as the Generalized Boltzmann Equation (GBE), first proposed by Larsen [2007] in the context of neutron transport (Section 3.3).

It is important to first clarify the difference between spatiallycorrelated media, and heterogeneous media, as commonly used in computer graphics. As illustrated in Figure 3, heterogeneous media assume local homogeneity at each differential volume $\mathrm{d} V(\mathbf{x})$. In contrast, correlated media take into account the average effect of uneven scatterer distributions within each $\mathrm{d} V(\mathbf{x})$. Therefore, a medium can be statistically homogeneous, meaning that its statistical moments are invariant over all the volume, but spatially correlated [Kostinski 2001].

\subsection{Background: The Radiative Transport Equation}

In its integro-differential form, the Radiative Transfer Equation (RTE) models the amount of radiance $L$ at point $\mathbf{x}$ in direction $\omega_{o}$ as:

$$
\omega_{o} \cdot \nabla L\left(\mathbf{x}, \omega_{o}\right)+\mu L\left(\mathbf{x}, \omega_{o}\right)=S\left(\mathbf{x}, \omega_{o}\right)+Q\left(\mathbf{x}, \omega_{o}\right),
$$
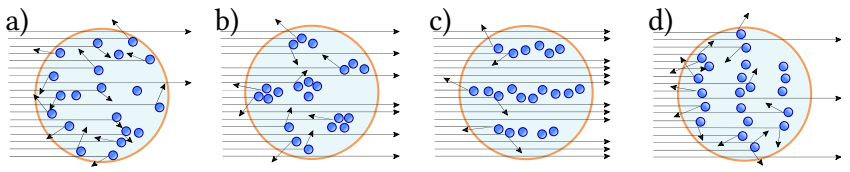

Fig. 4. Illustration of the effect of spatial correlation in a medium for different hypothetical distributions of scatterers. (a) A random distribution of scatterers within a differential volume $\mathrm{d} V(\mathrm{x})$. (b) In the presence of scatterer correlation, the probability of interaction changes within $\mathrm{d} V(\mathbf{x})$. (c, d) This correlation might further exhibit directional behavior, leading to very different interaction probabilities according to the degree of alignment with the propagation of light.

where $Q\left(\omega_{o}\right)$ is the volume source term, and $S$ is the in-scattered radiance:

$$
S\left(\mathbf{x}, \omega_{o}\right)=\mu_{s} \int_{\Omega} L\left(\mathbf{x}, \omega_{i}\right) f_{r}\left(\mathbf{x}, \omega_{i}, \omega_{o}\right) \mathrm{d} \omega_{i},
$$

which is the directional integral over the sphere $\Omega$ of the light scattered towards $\omega_{o}$, modeled using the phase function $f_{r} ; \omega_{i}$ represents the incoming direction of light. Note that we have omitted the spatial dependency of all terms in Equations (1) and (2) for simplicity. Finally, $\mu=\mu_{a}+\mu_{s}\left[\mathrm{~m}^{-1}\right]$ is the extinction coefficient, with $\mu_{a}$ and $\mu_{s}$ the absorption and scattering coefficients respectively. These terms model the probability of a beam of light to be attenuated either by absorption or scattering, and are defined as the product of the number of scatterers per unit volume $C\left[\mathrm{~m}^{-3}\right]$, and the scatterers' cross section $\sigma\left[\mathrm{m}^{2}\right]$, assuming that the scatterers are uniformly distributed in the differential volume (Figure 1, left) (see [Arvo 1993] for a detailed derivation). Jakob et al. [2010] later generalized the RTE to model directionally anisotropic media, by taking into account the angular (directional) dependence of the scatterers' cross section in media.

\subsection{Effect of Spatial Correlation on Extinction}

When light propagates through a participating medium, it scatters as a function of the distribution of the scatterers. When this distribution is random and uniformly distributed, extinction becomes a Poissonian process, and the exponential Beer-Lambert law accurately describes the attenuation of light (see [Gallavotti 1972] for a rigorous derivation). However, the distribution of scatterers in many media exhibits different forms and degrees of spatial correlation (e.g. clouds [Davis and Marshak 2004; Lovejoy et al. 1995], textiles [Coquard and Baillis 2006], porous materials [Bellet et al. 2009; Taine et al. 2010], or granular aggregates [Meng et al. 2015]). This affects light transport, as Figure 4 illustrates; as a consequence, attenuation is no longer exponential, and light extinction becomes non-Poissonian.

Negative correlation occurs when the distribution of scatterers is more uniform than Poisson (as in electrostatic repulsion), and leads to super-exponential (faster) extinction. Clustered scatterers, on the other hand, yield positive correlation, which leads to sub-exponential (slower) extinction; this is illustrated in Figure 5. The reason for such non-exponential transmittance can be further visualized intuitively in Figure 6: In negatively-correlated media, absorbers are less likely to "shadow" one another; as a result, more 


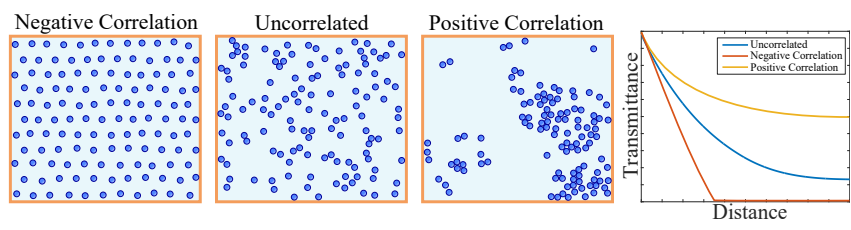

Fig. 5. Different types of scatterer correlation, and their effect on transmittance. From left to right, the first three figures depict negative correlation, no correlation (extinction is a Poissonian process), and positive correlation. The plot on the right shows extinction, averaged for several procedural realizations of the media (see Section S.11 in the supplemental) and ray directions: While uncorrelated media results in the classic exponential extinction, negative and positive correlation lead to faster- and slower-than-exponential extinction, respectively.

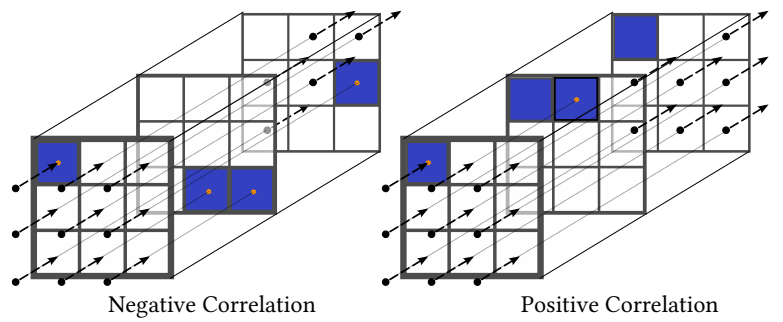

Fig. 6. Intuitive explanation for non-exponential transmittance in negatively(left) and positively-correlated (right) media. Solid squares represent perfect absorbers. Although both media have the same number of absorbers, shadowing (or overlapping) of such scatterers (positive correlation) results in less (sub-exponential) extinction. Figure inspired from [Kostinski 2002].

light becomes extinct. Positively-correlated media presents the opposite case, with many absorbers shadowing others; this creates empty regions which in turn lead to more light passing through.

More formally, in uncorrelated media (Poissonian process) the extinction probability after traveling a distance $t$ from the previous scattering (or emission ${ }^{2}$ ) event is $p(t)=\mu \exp (-\mu t)$, as predicted by the Beer-Lambert law. Thus, defining the differential probability of extinction $\Sigma(t)\left[\mathrm{m}^{-1}\right]$ as [Larsen and Vasques 2011]

$$
\Sigma(t)=\frac{p(t)}{1-\int_{0}^{t} p(s) \mathrm{d} s},
$$

where the denominator is the physical definition of transmittance $T(t)$, we obtain $\Sigma(t)=\mu$, the extinction coefficient of the medium (uniform for each differential volume, and independent of the distance $t$ ).

Let us now define a simple positively-correlated (clustered) medium, composed of regions with a high density of scatterers (extinction coefficient $\mu_{1}$ ), and regions with low density $\left(\mu_{2}\right)$. The probability of light extinction ${ }^{3}$ after traveling a distance $t$ is given by

$$
p(t)=\mu_{1} p_{\tau}\left(\mu_{1}\right) e^{-\mu_{1} t}+\mu_{2} p_{\tau}\left(\mu_{2}\right) e^{-\mu_{2} t},
$$

with $p_{\tau}\left(\mu_{1}\right)$ and $p_{\tau}\left(\mu_{2}\right)$ the probability of traversing a region with extinction coefficients $\mu_{1}$ and $\mu_{2}$ respectively, where $p_{\tau}\left(\mu_{1}\right)+p_{\tau}\left(\mu_{2}\right)=$

\footnotetext{
${ }^{2}$ We will refer only to scattering events from now on for simplicity.

${ }^{3}$ The probability of extinction $p(t)$ is also termed in the literature "path length distribution", "free path distribution", or "chord length distribution".
}

1. From this simple example, we can see that $p(t)$ is no longer exponential, and thus extinction is no longer a Poissonian process with a constant $\Sigma(t)=\mu$. Instead, plugging Equation (4) into Equation (3) leads to a function dependent on $t$. In other words, spatial correlation introduces a memory effect [Kostinski 2002], where the differential probability of extinction depends on the traveled distance $t$ since the previous scattering event. This has a significant effect in the final volumetric appearance of the medium, as shown in Figure 1 and throughout this paper.

\subsection{The Generalized Boltzmann Equation}

Since $\Sigma(t)$ is a function of $t$ in the presence of correlation, we need to introduce the $t$-dependent flux $L\left(\mathbf{x}, \omega_{o}, t\right)\left[\mathrm{W} \mathrm{m}^{-2} \mathrm{sr}^{-1} \mathrm{~m}^{-1}\right]$, the flux at $\mathbf{x}$ after traveling a distance $t$ from the last scattering event. It relates with classic flux $L\left(\mathbf{x}, \omega_{o}\right)\left[\mathrm{W} \mathrm{m}^{-2} \mathrm{sr}^{-1}\right]$ as $L\left(\mathbf{x}, \omega_{o}\right)=$ $\int_{0}^{\infty} L\left(\mathbf{x}, \omega_{o}, t\right) \mathrm{d} t$, and in turn introduces an additional derivative term in Equation (1), resulting in the Generalized Boltzmann Equation [Larsen 2007; Larsen and Vasques 2011]

$$
\begin{gathered}
\frac{d}{d t} L\left(\mathbf{x}, \omega_{o}, t\right)+\omega_{o} \cdot \nabla L\left(\mathbf{x}, \omega_{o}, t\right)+\Sigma(t) L\left(\mathbf{x}, \omega_{o}, t\right)=0, \\
L\left(\mathbf{x}, \omega_{o}, 0\right)=\underbrace{\int_{0}^{\infty} \Sigma_{s}(t) \int_{\Omega} L\left(\mathbf{x}, \omega_{i}, t\right) f_{r}\left(\omega_{i}, \omega_{o}\right) \mathrm{d} \omega_{i} \mathrm{~d} t}_{\text {Inscattering } S\left(\mathbf{x}, \omega_{o}\right)}+Q\left(\mathbf{x}, \omega_{o}\right),
\end{gathered}
$$

where $\Sigma_{S}(t)=\Lambda \Sigma(t)$ is the probability of a photon being scattered after traveling a distance $t$ (see Section S.1 in the supplemental for the full derivation), and $\Lambda$ represents albedo. The second line of the equation represents the value for $t=0$, in which light is scattered or emitted. Thus, after each scattering event the memory effect for the extinction is reset to zero.

As expected, by removing the $t$-dependency as $\Sigma(t)=\Sigma$, and integrating Equation (5), we obtain the classic RTE [Equation (1)] (see Section S.2 in the supplemental). Moreover, Equation (5) can also support directionally anisotropic media [Jakob et al. 2010] by formulating $\Sigma$ as a function of $\omega_{o}$ [Vasques and Larsen 2014].

\section{OUR EXTENDED GBE}

\subsection{Limitations of the GBE}

Unfortunately, Equation (5) relies on a set of simplifying assumptions, which limit its applicability in rendering applications. In particular [Larsen 2007; Larsen and Vasques 2011]: ${ }^{4}$

(1) The medium is statistically homogeneous, and infinite; no system boundaries exist.

(2) The phase function $f_{r}\left(\omega_{i}, \omega_{o}\right)$ and albedo $\Lambda$ are independent of $t$. For example, in a mixture of two types of scatterers with different phase function or albedo, this assumes that both types have the same structure.

(3) The source term $Q\left(\mathbf{x}, \omega_{o}\right)$ is correlated with the scatterers in the volume. This assumption does not hold in most cases, as illustrated in Figure 7.

\footnotetext{
${ }^{4}$ Larsen and Vasques also assume a monoenergetic system; for simplicity, we assume also a single wavelength, although removing this limitation is straight forward.
} 

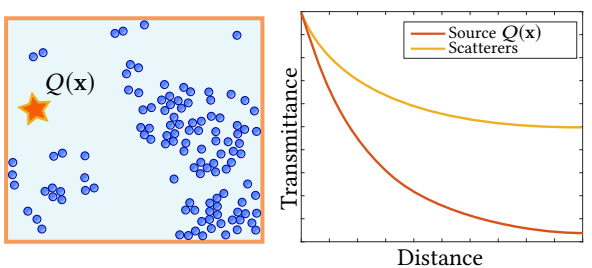

Fig. 7. Left: An uncorrelated light source $Q(\mathrm{x})$ in a positively-correlated medium. The differential probability of extinction $\Sigma(\mathrm{x}, t)$ is therefore different for each, which significantly modifies light transport, as shown in the right, where transmittance is numerically computed from several procedurally generated media with identical positive correlation. This difference is not captured in Larsen's original GBE [Larsen and Vasques 2011].

In order to make the GBE useful for rendering, we need to extend it beyond these limiting assumptions. We describe this in the rest of this section, introducing our novel Extended GBE.

\subsection{Extending the GBE}

To lift the first and second limitations of the standard GBE, we first reformulate $\Sigma, \Lambda$, and $f_{r}$ as functions of the spatial position and the traveled distance, as $\Sigma(\mathbf{x}, t), \Lambda(\mathbf{x}, t)$, and $f_{r}\left(\mathbf{x}, \omega_{i}, \omega_{o}, t\right)$ respectively. This means that, depending on the traveled distance $t$, light will be scattered differently, according to the different spatial correlation of the scatterers. Note that some previous works in graphics [Frisvad et al. 2007; Sadeghi et al. 2012] have included a mixture of scatterer sizes in the medium, but not spatial correlation. Defining the directional scattering operator $\mathrm{B}\left(\mathrm{x}, \omega_{i}, \omega_{o}, t\right)=$ $\Lambda(\mathbf{x}, t) \Sigma(\mathbf{x}, t) f_{r}\left(\mathbf{x}, \omega_{i}, \omega_{o}, t\right)$ for compactness, Equation (5) becomes

$$
\begin{gathered}
\frac{d}{d t} L\left(\mathbf{x}, \omega_{o}, t\right)+\omega_{o} \cdot \nabla L\left(\mathbf{x}, \omega_{o}, t\right)+\Sigma(t) L\left(\mathbf{x}, \omega_{o}, t\right)=0, \\
L\left(\mathbf{x}, \omega_{o}, 0\right)=\int_{0}^{\infty} \int_{\Omega} L\left(\mathbf{x}, \omega_{i}, t\right) \mathrm{B}\left(\mathbf{x}, \omega_{i}, \omega_{o}, t\right) \mathrm{d} \omega_{i} \mathrm{~d} t+Q\left(\mathbf{x}, \omega_{o}\right),
\end{gathered}
$$

where we assume an isotropic formulation to avoid cluttering.

Lifting the third assumption, on the other hand, requires a more significant change of Equation (5). In Larsen's original formulation of the GBE, since $L\left(\mathbf{x}, \omega_{o}, 0\right)$ includes both scattering $S\left(\mathbf{x}, \omega_{o}\right)$ and light emitted by sources $Q\left(\mathbf{x}, \omega_{o}\right)$, both terms implicitly share the same differential probability of extinction $\Sigma(\mathbf{x}, t)$. However, this would only be true if they present the exact same correlation (e.g. the scattering and the emissive particles are the same); in the general case, $\Sigma(\mathbf{x}, t)$ is different for $S$ and $Q$. Moreover, different sources $Q$ might correlate differently with the medium, leading to different $\Sigma(\mathbf{x}, t)$ per source. Figure 7 shows how having different $\Sigma(\mathbf{x}, t)$ for scatterers and sources significantly affects light transport. This different correlation between sources and scatterers is in fact very important for rendering since, as we show later in Section 4.3, reflections at media boundaries act as uncorrelated sources.

Taking all this into account, we can express radiance $L\left(\mathbf{x}, \omega_{\mathbf{o}}, t\right)$ as

$$
L\left(\mathbf{x}, \omega_{o}, t\right)=L_{S}\left(\mathbf{x}, \omega_{o}, t\right)+\sum_{j} L_{Q_{j}}\left(\mathbf{x}, \omega_{o}, t\right),
$$

where $L_{S}\left(\mathbf{x}, \omega_{\boldsymbol{o}}, t\right)$ is the scattered radiance reaching $\mathbf{x}$ after traveling a distance $t$ since the last scattering event, and $L_{Q_{j}}\left(\mathbf{x}, \omega_{o}, t\right)$ is the unscattered radiance directly emitted by source $Q_{j}$, which has traveled a distance $t$ since emission. We can then transform Equation (6) into our Extended GBE as

$$
\begin{aligned}
\frac{d}{d t} L\left(\mathbf{x}, \omega_{o}, t\right) & +\omega_{o} \cdot \nabla L\left(\mathbf{x}, \omega_{o}, t\right)+\Sigma_{S}(\mathbf{x}, t) L_{S}\left(\mathbf{x}, \omega_{o}, t\right) \\
& +\sum_{j} \Sigma_{Q_{j}}(\mathbf{x}, t) L_{Q_{j}}\left(\mathbf{x}, \omega_{o}, t\right)=0
\end{aligned}
$$

where $\Sigma_{S}(\mathbf{x}, t)$ and $\Sigma_{Q_{j}}(\mathbf{x}, t)$ are the differential extinction probabilities for the scattered photons and the (unscattered) photons emitted by light source $Q_{j}$, respectively. Then, for $t=0$ we have

$$
\begin{aligned}
L_{S}\left(\mathbf{x}, \omega_{o}, 0\right)=\int_{0}^{\infty} & \int_{\Omega}\left(\mathrm{B}_{S}\left(\mathbf{x}, \omega_{i}, \omega_{o}, t\right) L_{S}\left(\mathbf{x}, \omega_{i}, t\right)\right. \\
& \left.+\sum_{j} \mathrm{~B}_{Q_{j}}\left(\mathbf{x}, \omega_{i}, \omega_{o}, t\right) L_{Q_{j}}\left(\mathbf{x}, \omega_{i}, t\right)\right) \mathrm{d} \omega_{i} \mathrm{~d} t \\
L_{Q_{j}}\left(\mathbf{x}, \omega_{o}, 0\right) & =Q_{j}\left(\mathbf{x}, \omega_{o}\right)
\end{aligned}
$$

where $\mathrm{B}_{S}\left(\mathbf{x}, \omega_{i}, \omega_{o}, t\right)=\Lambda_{S}(\mathbf{x}, t) \Sigma_{S}(\mathbf{x}, t) f_{r, S}\left(\mathbf{x}, \omega_{i}, \omega_{o}, t\right)$ is the scattering operator for scattered photons (thus representing a multiple scattering operator), and $\mathrm{B}_{Q_{j}}\left(\mathbf{x}, \omega_{i}, \omega_{o}, t\right)$ is the scattering operator for photons emitted by light source $Q_{j}$ (single scattering operator). Note how, interestingly, Equation (9) makes the convenient separation between multiple and single scattering explicit. Similar to $\Sigma(\mathbf{x}, t)$, the phase function and albedo terms might also be different, depending on the correlation between sources and the scatterers. It is easy to verify that when the sources and scatterers are equally correlated with the rest of the medium, the Extended GBE in Equation (8) simplifies to Equation (5) (see Section S.3 in the supplemental).

Integral form of the Extended GBE. In order to get an integral formulation of our Extended GBE usable in a general Monte Carlo renderer, we solve Equation (8) for the incoming radiance at point $\mathbf{x}$ as (see Section S.4 in the supplemental for the full derivation)

$$
\begin{aligned}
L\left(\mathbf{x}, \omega_{o}\right)=\int_{0}^{\infty} & T_{S}\left(\mathbf{x}, \mathbf{x}_{t}\right) S\left(\mathbf{x}_{t}, \omega_{o}\right) \\
& +\sum_{j} T_{Q_{j}}\left(\mathbf{x}, \mathbf{x}_{t}\right) Q_{j}\left(\mathbf{x}_{t}, \omega_{o}\right) \mathrm{d} t
\end{aligned}
$$

where $\mathbf{x}_{t}=\mathbf{x}-\omega_{o} t$. The terms $T_{S}\left(\mathbf{x}, \mathbf{x}_{t}\right)=e^{-\int_{0}^{t} \Sigma_{S}(\mathbf{x}, s) \mathrm{d} s}$ and $T_{Q_{j}}\left(\mathbf{x}, \mathbf{x}_{t}\right)=e^{-\int_{0}^{t} \Sigma_{Q_{j}}(\mathbf{x}, s) \mathrm{d} s}$ represent transmittance between $\mathbf{x}$ and $\mathrm{x}_{t}$ for the scattered and emitted radiance, respectively. Last, $S\left(\mathbf{x}_{t}, \omega_{o}\right)$ is

$$
\begin{aligned}
S\left(\mathbf{x}_{t}, \omega_{o}\right) & =\int_{0}^{\infty} \int_{\Omega}\left(\mathrm{B}_{S}\left(\mathbf{x}_{t^{\prime}}, \omega_{i}, \omega_{o}, t^{\prime}\right) S\left(\mathbf{x}_{t^{\prime}}, \omega_{i}\right) T_{S}\left(\mathbf{x}_{t}, \mathbf{x}_{t^{\prime}}\right)\right. \\
& \left.+\sum_{j} \mathrm{~B}_{Q_{j}}\left(\mathbf{x}_{t^{\prime}}, \omega_{i}, \omega_{o}, t^{\prime}\right) Q_{j}\left(\mathbf{x}_{t^{\prime}}, \omega_{o}\right) T_{Q_{j}}\left(\mathbf{x}_{t}, \mathbf{x}_{t^{\prime}}\right)\right) \mathrm{d} \omega_{i} \mathrm{~d} t^{\prime},
\end{aligned}
$$




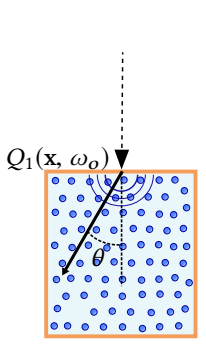

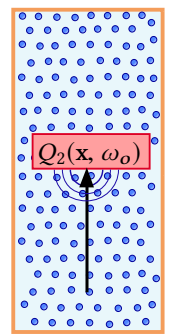

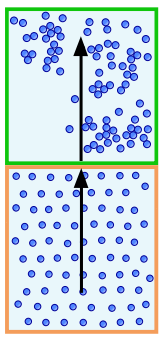

c)
Fig. 8. Schematic example of the different boundary conditions: a) light entering a medium (Vacuum to Medium); b) light being reflected from a boundary back into the medium (Medium to Surface); and c) light crossing the interface between two different media (Medium to Medium). Refer to the text for details.

where $\mathbf{x}_{t^{\prime}}=\mathbf{x}_{t}+\omega_{i} t^{\prime}$. Next, we describe boundary conditions, and how they affect light transport.

\subsection{Boundary Conditions}

The assumption that the medium is infinite and homogeneous ignores changes in correlation that occur at boundaries, such as photons entering a medium, the presence of surfaces inside, or the interface between two different media. Figure 8 illustrates the different boundary conditions and their effects in light transport. Here, we describe them and show how to incorporate them to our model.

Vacuum to Medium (Figure 8a): This is the simplest case, where an uncorrelated photon (from an uncorrelated medium or the vacuum) enters a correlated medium. It can be modeled as a source $Q_{1}\left(\mathbf{x}, \omega_{0}\right)$ at the entry boundary point $\mathbf{x}$, with $t=0$.

Medium to Surface (Figure 8b): This case accounts for the interaction with surfaces such as a dielectric boundary, or an object placed inside the medium. Such surfaces are uncorrelated with respect to the medium. We can model this as a new source $Q_{2}\left(\mathbf{x}, \omega_{\boldsymbol{o}}\right)=$ $L_{S}\left(\mathbf{x}, \omega_{i}\right) f\left(\mathbf{x}, \omega_{i}, \omega_{o}\right)$, with $L_{S}\left(\mathbf{x}, \omega_{i}\right)$ and $f\left(\mathbf{x}, \omega_{i}, \omega_{o}\right)$ the incoming radiance at $\mathrm{x}$ and the BSDF respectively, and setting $t=0$.

Medium to Medium (Figure 8c): A photon crosses the interface between two different homogeneous media of different structure and correlation (this boundary condition therefore enables modeling heterogeneous media as well). The probability of extinction in the second medium $p_{2}(t)$ depends not only on its correlation $\eta_{2}$ and the correlation of the first medium $\eta_{1}$, but also on the correlation between the two media $\eta_{1,2}$.

Figure 9 shows results for all three boundary conditions; please refer to Section S.11.3 in the supplemental for a more exhaustive set of examples.

\section{RENDERING WITH THE EXTENDED GBE}

Up to this point, we have extended Larsen's original GBE formulation [Equation (5)], lifting the assumptions that made it unsuitable for rendering, presenting it also in integral form. Our Extended GBE [Equations (8) and (11)] supports an arbitrary mixture of scatterers (see Appendix A), and accounts for the effect of different correlation between scatterers and sources. To use it for rendering, the required differential extinction probabilities can be tabulated [Frank and Goudon 2010; Larsen and Vasques 2011] by simulating via Monte Carlo an estimate of $p(t)$, based on an explicit representation of the volume. This is a similar approach to our numerical results in Section S.11 in the supplemental, and the validation curves computed by Meng et al. [2015, Figure 6] to validate their uncorrelated radiative transfer-based approximation. Alternatively, an empirical $p(t)$ can be used, fitting the observed transmittance in experimental setups, as is common in atmospheric sciences [Davis et al. 1999]. In both cases, $\Sigma(\mathbf{x}, t)$ is computed by inverting $p(t)$ via Equation (3).

In computer graphics, participating media are usually described in terms of their optical parameters. However, in our current formulation of the Extended GBE, there is no explicit connection with such parameters. In the following, we provide the missing connection: We formulate a model for correlated media based on the optical parameters commonly used in rendering, which is intuitive to use and easy to plug into our Extended GBE. Then, we propose a simplified version of the model based on the assumption of positive correlation, which is easy to use and efficient to sample and evaluate.

\subsection{Modeling Correlated Media from Optical Parameters}

In a rendering context, the optical properties of a participating medium (e.g. extinction coefficients, scattering albedo, or phase function) are usually defined locally. Unfortunately, at the heart of our Extended GBE [Equation (8)] lies the differential extinction probability $\Sigma(\mathbf{x}, t)$, whose memory effect depends on the spatial correlation at neighboring points, and thus cannot be defined locally. Our goal then is to model $\Sigma(\mathbf{x}, t)$ and its derived quantities $p(t)$ and $T(t)$, based on probability distributions of extinction $p_{\tau}(\mu)$. In the following, we assume both homogeneity in the neighborhood of $\mathbf{x}$ and isotropy, so we remove the spatial and angular dependence from the following derivations for clarity.

Given a ray $r$ in a medium, we can define its input radiance as $L_{i}(\mathrm{r})$, and its attenuation as $\mathcal{T}\left(\tau_{t}(\mathrm{r})\right)$, the ratio of input and output radiance of a single ray $r$ defined as a probabilistic function, which depends on the ray's optical depth $\tau_{t}(\mathfrak{r})$. Considering now a beam of light $\mathcal{R}$ composed of several parallel rays $\mathfrak{r} \in \mathcal{R}$ (see Figure 10), the total radiance $L_{o}(t)$ traveling a distance $t$ in a correlated medium can be expressed as

$$
L_{o}(t)=\int_{\mathcal{R}} L_{i}(\mathrm{r}) \mathcal{T}\left(\tau_{t}(\mathfrak{r})\right) \mathrm{d} \mathfrak{r}
$$

In granular media [Moon et al. 2007], where the correlation length is larger than a differential distance $\mathrm{d} t$ (and usually larger than the granular particle's size), the probability of extinction $p(t)$ depends on the distribution of scatterers along the direction of propagation of light, and needs to be taken into account explicitly. However, local correlation is assumed to be smaller than $\mathrm{d} t$; this means that the exact position of the scatterers within the volume becomes irrelevant, and only their projection onto the plane $\mathcal{P}$ perpendicular to the propagation direction beam $\mathcal{R}$ matters. We can then simplify the expression for the optical depth ${ }^{5}$ to the homogeneous case

\footnotetext{
5 "Optical depth" is a standard term in physics, defined as the natural logarithm of the ratio of incident to transmitted radiant power through a material.
} 

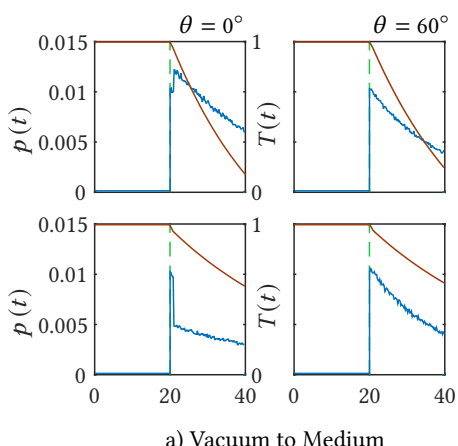

a) Vacuum to Medium
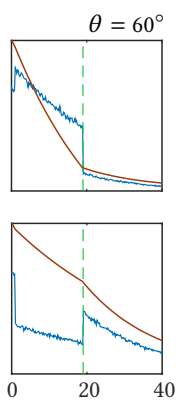

b) Medium to Surface
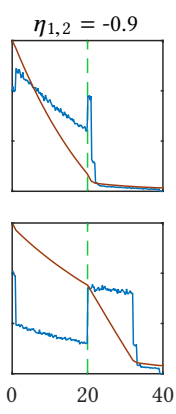

40
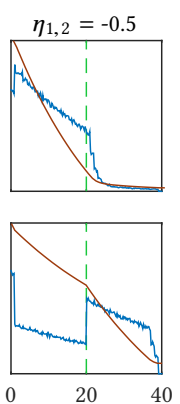
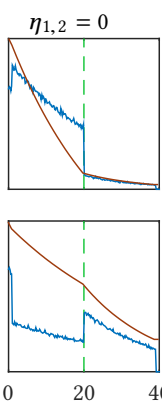

c) Medium to Medium
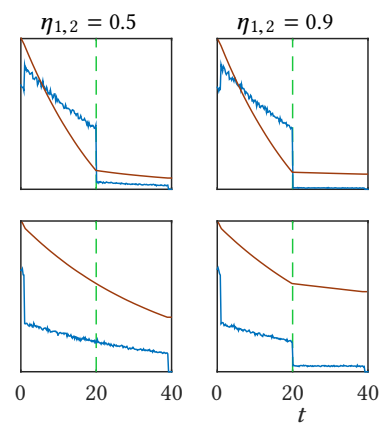

40

Fig. 9. Probability of extinction $p(t)$ (blue) and transmittance $T(t)$ (orange) as a function of $t$, for example cases of our three different boundary conditions. The vertical dotted line indicates the boundary. The first medium has a negative correlation $\eta_{1}=-0.5$ on the top row, and positive $\eta_{1}=0.5$ on the bottom row. (a) Vacuum to Medium. When light enters the medium, it acts as a source term $Q\left(\mathbf{x}, \omega_{o}\right)$, which depends on the angle of incidence $\theta$, since correlation might present some directionality (see Figure 8). (b) Medium to Surface. As light is reflected on a surface boundary and changes direction, it acts as a directionally-resolved source $Q\left(\mathbf{x}, \omega_{o}\right)$ which depends on the surface BSDF. c) Medium to Medium, for a varying correlation $\eta_{1,2}=[-0.9,0.9]$ between the two media. For increasingly positive correlation $\eta_{1,2}$ (high probability of the first medium shadowing the second), $p_{2}(t)$ becomes lower. For increasingly negative correlation $\eta_{1,2}$ (low shadowing probability), $p_{2}(t)$ becomes higher near the boundary (then depends on $\left.\eta_{2}\right)$. For uncorrelated media $\left(\eta_{1,2}=0\right)$, incoming photons can be modeled as sources at the entry boundary points, with $Q\left(\mathbf{x}, \omega_{o}\right)$ dependent on the correlation of the second medium $\eta_{2}$. For all cases modeled as light sources $Q\left(\mathbf{x}, \omega_{o}\right), t$ is set to 0 . Refer to the supplemental material for a more comprehensive set of examples.

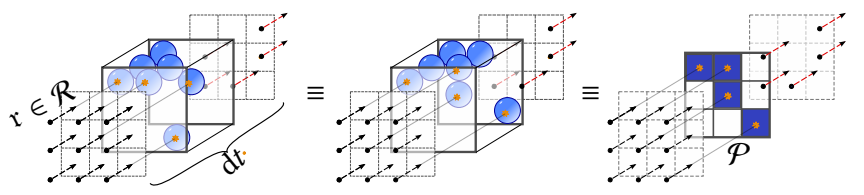

Fig. 10. Left and center: Examples of two differential volumes in a medium, each with different distributions of scatterers, but with a similar projection on the plane perpendicular to the direction of propagation (right).

where $\tau_{t}(\mathfrak{r})=\mu(\mathfrak{r}) t$, with $\mu(\mathfrak{r})$ the density of scatterers found by an individual ray $r$ when traversing the medium.

However, explicitly integrating over all rays in $\mathcal{R}$ is not practical. Instead, we would like to find a compact way of relating $L_{i}(\mathrm{r})$ to the extinction coefficient $\mu(\mathfrak{r})$. We can remove its dependence on ray $\mathfrak{r}$ by modeling $L_{i}$ as a probability distribution $p_{L}\left(L_{i}\right)$ (e.g. by taking the histogram of $\left.L_{i}(\mathrm{r})\right)$, and explicitly relating it with the extinction coefficient $\mu$ via a conditional probability distribution $p_{\tau}\left(\mu ; L_{i}\right)$. We therefore transform Equation (13) into

$$
L_{o}(t)=\int_{0}^{\infty} \int_{0}^{\infty} p_{L}\left(L_{i}\right) p_{\tau}\left(\mu ; L_{i}\right) L_{i} \mathcal{T}(\mu t) \mathrm{d} \mu \mathrm{d} L_{i} .
$$

Defining $\hat{L}_{i}=\int_{\mathcal{R}} L_{i}(\mathrm{r}) \mathrm{dr}=\int_{0}^{\infty} p_{L}\left(L_{i}\right) L_{i} \mathrm{~d} L_{i}$, and using $T(t)=$ $\frac{L_{o}(t)}{\hat{L}_{i}}$ we get

$$
T(t)=\int_{0}^{\infty} \int_{0}^{\infty} p_{L}\left(L_{i}\right) p_{\tau}\left(\mu ; L_{i}\right) \frac{L_{i}}{\hat{L}_{i}} \mathcal{T}(\mu t) \mathrm{d} \mu \mathrm{d} L_{i},
$$

which models transmittance $T(t)$ as a function of the correlation between the light and the distribution of local scatterers. Finally, from Equation (15) we can compute the probability of extinction as $p(t)=\left|\frac{\mathrm{d} T(t)}{\mathrm{d} t}\right|$, while $\Sigma(t)$ can be obtained as $\Sigma(t)=p(t) / T(t)$ [Equation (3)].

\subsection{An intuitive local model for positively-correlated media}

Equation (15) is general and can model any type of correlation; for the common case of positive correlation, we can set $\mathcal{T}(\mu t)=e^{-\mu t}$ (see [Kostinski 2002] for details), and assume that $p_{L}\left(L_{i}\right)$ and $p_{\tau}(\mu)$ are independent, so that $p_{\tau}\left(\mu ; L_{i}\right)=p_{\tau}(\mu)$. We can then rewrite Equation (15) as (see Section S.5 in the supplemental)

$$
T(t)=\int_{0}^{\infty} e^{-\mu t} p_{\tau}(\mu) \mathrm{d} \mu .
$$

Using again the relationship in Equation (3), we obtain the differential extinction probability

$$
\Sigma(t)=\frac{p(t)}{T(t)}=\frac{\int_{0}^{\infty} \mu e^{-\mu t} p_{\tau}(\mu) \mathrm{d} \mu}{\int_{0}^{\infty} e^{-\mu t} p_{\tau}(\mu) \mathrm{d} \mu} .
$$

Note that this form of $p(t)$ [numerator in Equation (17)] is a generalization of the simple example in Equation (4) for a mixture of two different extinction coefficients. Assuming that the light distribution $p_{L}\left(L_{i}\right)$ from both sources $Q_{j}$ and scatterers $S$ is uncorrelated with the scatterers distribution $p_{\tau}(\mu)$, then $\Sigma(t)=\Sigma_{S}(t)=\Sigma_{Q_{j}}(t)$.

Finding a good distribution $p_{\tau}(\mu)$. To be able to use Equations (16) and (17), we need to find a good optical depth distribution $p_{\tau}(\mu)$ for the medium. Taking the average scatterers' cross section $\sigma$, we can define

$$
\mu p_{\tau}(\mu)=C p_{C}(C) \sigma
$$

where $C$ is the scatterers concentration and $p_{C}(C)$ its probability distribution. To find a practical $p_{C}(C)$ we analyzed a wide range of high-resolution volumes exhibiting different correlation (see Figure 11 for some examples). We observed that a gamma distribution fits $p_{C}(C)$ reasonably well, so that

$$
p_{C}(C) \approx \Gamma(C ; \alpha, \beta)=\frac{\beta^{\alpha} C^{\alpha-1} e^{-C \beta}}{\gamma(\alpha)},
$$



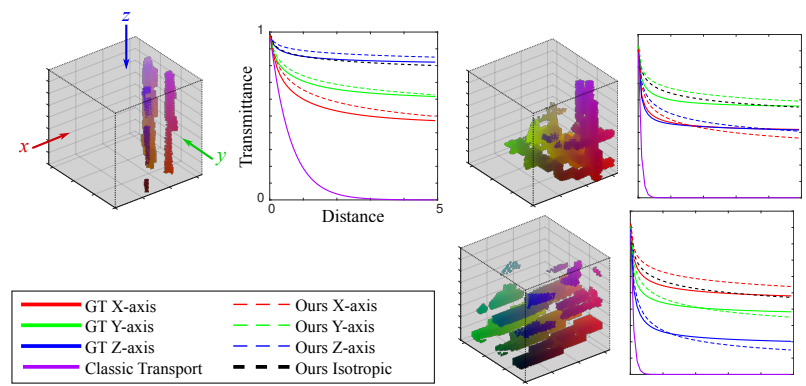

Fig. 11. Transmittance in high-resolution volumes of locally-correlated media (procedurally generated after [Lopez-Moreno et al. 2015]). Beams of light travel through each volume, aligned in succession to the $x, y$, and $z$ axes. Ground-truth transmittance (red, green, and blue solid lines) has been computed by brute force regular tracking [Amanatides and Woo 1987], while our simulation (dotted lines) uses the gamma distribution proposed in Equation 19. Classic transport governed by the RTE significantly overestimates extinction through the volume, resulting in an exponential decay (purple line). In contrast, our model matches ground-truth transmission much more closely. The black dotted line is the result of isotropic correlation, which is clearly also non-exponential. Please refer to Figure S.13 in the supplemental for more examples.

with $\alpha=\bar{C}^{2} \cdot \operatorname{Var}(C)^{-1}, \beta=\bar{C} \cdot \operatorname{Var}(C)^{-1}$, and $\gamma(\alpha)$ the gamma function. Moreover, previous research has shown that the gamma distribution is also very adequate for modeling the concentration of turbulent media such as clouds [Barker et al. 1996], or particulate media [Peltoniemi and Lumme 1992].

Equation (19) provides a compact and intuitive description of the statistical properties of $p_{C}(C)$ [and in turn of $p_{\tau}(\mu)$ in Equation (18)], by only using its mean $\bar{C}$ and variance $\operatorname{Var}(C)$ (intuitively, a higher variance indicates clusters of scatterers with gaps between them). In contrast, traditional (uncorrelated) media depend only on the mean concentration $\bar{C}$, and assume $\operatorname{Var}(C)=0$. For simplicity, we have assumed that both $p_{C}(C)$ and $\sigma$ are isotropic. Appendix B shows how to add directional dependencies as $p_{C}\left(C ; \omega_{o}\right)$ and $\sigma\left(\omega_{o}\right)$.

Rendering. Using Equations (19) and (16), and noting that the latter is related with the moment distribution function $M(t)$ of $p_{C}(C)$ as $T(t)=M(-\sigma t)$ [Davis and Xu 2014], we can compute the transmittance, probability of extinction, and differential probability of extinction as

$$
\begin{aligned}
& T(t)=\left(1+\frac{\sigma}{\beta} t\right)^{-\alpha}, \\
& p(t)=\frac{\alpha \sigma}{\beta}\left(1+\frac{\sigma}{\beta} t\right)^{-(1+\alpha)}, \\
& \Sigma(t)=\frac{\alpha \sigma}{\beta}\left(1+\frac{\sigma}{\beta} t\right)^{-1} .
\end{aligned}
$$

In Figure 11 we analyze the performance of our analytic expression of transmittance for correlated media in Equation (20), against the exponential transmittance predicted by the Beer-Lambert law, and ground-truth transmittance computed by brute force regular tracking [Amanatides and Woo 1987]. Our model is much closer to the ground truth than the result of classic light transport, which significantly overestimates extinction through the volume. Figure 12
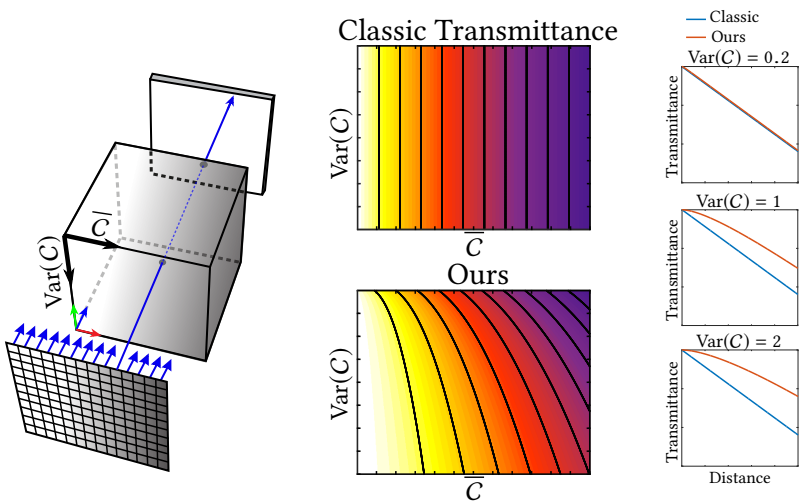

Fig. 12. Comparison between traditional transmittance as predicted by the Beer-Lambert law, and our transmittance for correlated media. The scene consists of a cube embedding a participating medium, placed in front of a light source. The medium has a constant cross section $\sigma=1$, and increasing scatterers concentration $\bar{C}$ and correlation (i.e. density variance $\operatorname{Var}(C)$ ) along the horizontal and vertical axes, respectively. Correlation does not affect transmittance in the classic model, which follows the Beer-Lambert law as shown in the log-scale plots on the right. In contrast, our model captures the slower-than-exponential decay as variance $\operatorname{Var}(C)$ increases. We use $\bar{C}=1$ for the plots. Figure after [Novák et al. 2014].

explores our closed-form of transmittance: As variance increases, the slower-than-exponential behavior becomes more pronounced, as observed by Davis and Mineev-Weinstein [2011] when analyzing the frequency of density fluctuations in correlated media. This effect is not captured by classic light transport.

In a Monte Carlo renderer, we can compute a random walk by sampling transmittance using the probability defined in Equation (21). However, as opposed to the classic exponential transmittance in the Beer-Lambert law, $p(t)$ is not proportional to $T(t)$, which may lead to increase the variance of the estimate. To sample with a probability $p(t) \propto T(t)$, assuming $\alpha>1$ (i.e. $\bar{C}>\sqrt{(} \operatorname{Var}(C))$ we can define $p(t)$ as

$$
p(t)=-\sigma \frac{1-\alpha}{\beta}\left(1+\frac{\sigma}{\beta} t\right)^{-\alpha}=-\sigma \frac{1-\alpha}{\beta} T(t),
$$

which can be sampled using its inverse cdf

$$
t(\xi)=-\frac{\beta}{\sigma}(1-\sqrt[1-\alpha]{1-\xi})
$$

with $\xi \in[0,1]$ a uniform random value. When the sampled distance $t$ is longer than the distance $t^{\prime}$ to a boundary condition, the probability of an intersection at $t^{\prime}$ becomes

$$
p\left(t^{\prime}\right)=\left(1+\frac{\sigma}{\beta} t^{\prime}\right)^{1-\alpha} .
$$

We refer to Section S.7 in the supplemental for more detailed derivations, including the general case where $\alpha>0$.

Implementation. While correlated media can be implemented as a volumetric definition in most renderers, there are a few details that need to be taken into account. The most important one is that the constants used when solving the classic RTE (e.g. $\Lambda$ or $\Sigma(t)=\mu$ ) are now defined as a function of $t$. As such, most of the optimizations 

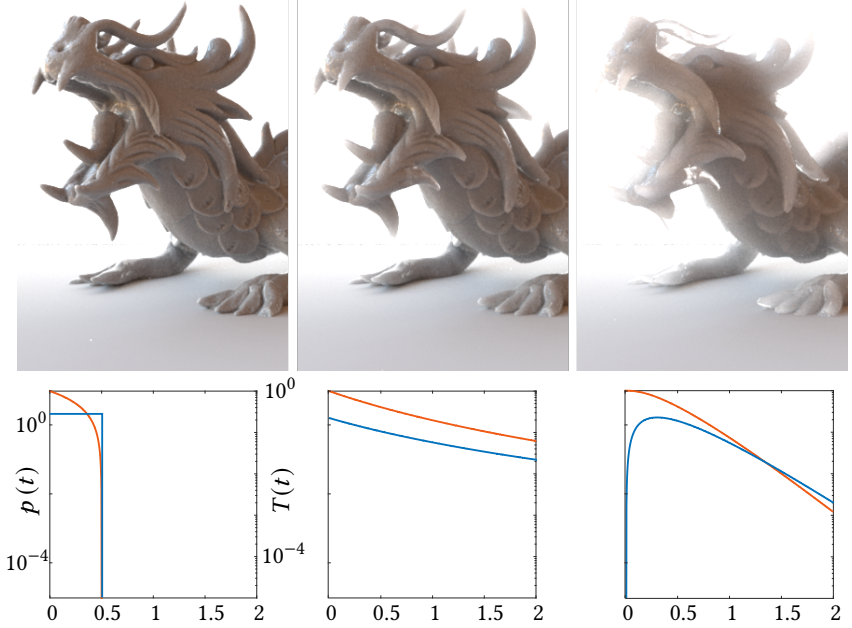

Fig. 13. Materials with different types of probability distributions of extinction $p(t)$ (shown in the the bottom plots in blue, while transmittance $T(t)$ is shown in orange; both cases are in log-scale). From left to right: Negative correlation with linear extinction; a power-law $p(t)$ resulting from our local model (Section 5.2), with $\operatorname{Var}(C(\mathrm{x}))=1$; and an example of one empirical $p(t)$ following a gamma distribution with $\operatorname{Var}(t)=.1$ (see Section S.8 in the supplemental for details). In all cases we use mean extinction $\bar{\mu}=2 \mathrm{~m}^{-1}$, albedo $\Lambda=.8$, and isotropic phase function.

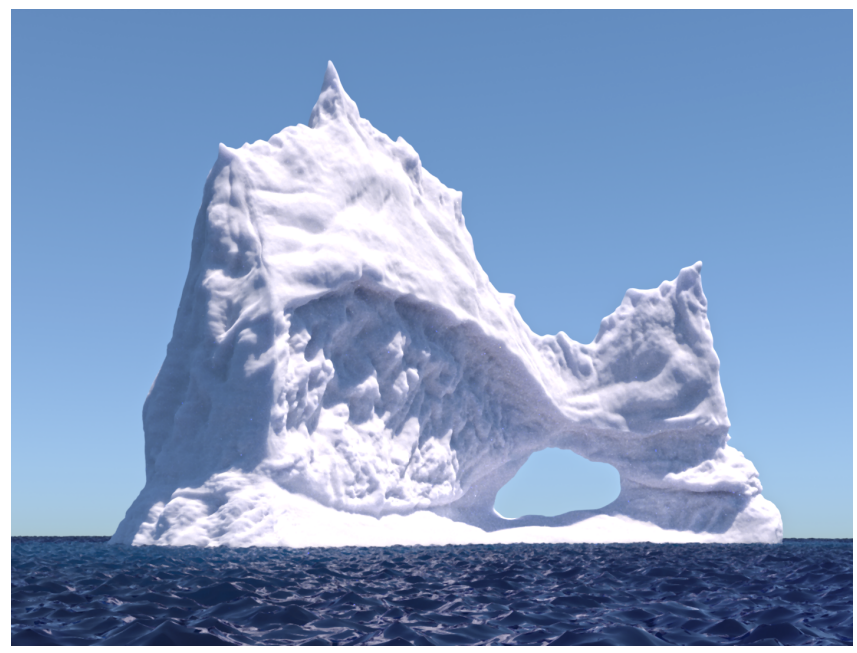

Fig. 14. Rendering of an iceberg made of compacted snow (with snow's spectral cross section $\sigma$ and albedo $\Lambda$ after Frisvad and colleagues [2007]), using our model in Section 5.2.

typically done in the photon's random walk due to terms cancellation cannot be directly applied here. Additionally, the different correlations between scatterers and sources in Equation (11) require keeping track of the previous vertex of the path when sampling a new one (via selecting either $p_{Q}(t)$ or $p_{S}(t)$ ). This is also important when connecting with the light source via next-event estimation, where the source's transmittance and differential scattering probability need to be applied.
Table 1. Computational cost for the images shown in the paper, for both uncorrelated (traditional model) and correlated media (ours). When different types of correlation are used, we show two measurements (positive / negative).

\begin{tabular}{cccc}
\hline Figure & \# Samples & Uncorrelated & Correlated \\
1 & 4096 & $53 \mathrm{~m}$ & $45 \mathrm{~m} / 58 \mathrm{~m}$ \\
13 & 4096 & $30 \mathrm{~m}$ & $33 \mathrm{~m} / 35 \mathrm{~m}$ \\
14 & 2048 & $185 \mathrm{~m}$ & $213 \mathrm{~m}$ \\
15 & 4096 & $26 \mathrm{~m}$ & $28 \mathrm{~m}$ \\
16 & 2048 & $5.6 \mathrm{~m}$ & $5.8 \mathrm{~m}$ \\
17 & 8192 & $70 \mathrm{~m}$ & $82 \mathrm{~m}$ \\
18 & 4096 & $17.71 \mathrm{~m}$ & $18.8 \mathrm{~m}$ \\
\hline
\end{tabular}

\section{RESULTS}

In this section we show results using our new model for spatiallycorrelated participating media, including comparisons against the traditional RTE. We have implemented the integral form of our Extended GBE [Equation (11)] as a volumetric definition in Mitsuba [Jakob 2010]. For materials with negative correlation we have used a linear transmittance decay (see Section S.8 in the supplemental for details); for positive correlation, we used our local model in Section 5.2. Unless stated otherwise, we assume positivelycorrelated media in our results. All our tests were performed on an Intel Core i7-6700K at $4 \mathrm{GHz}$ with $16 \mathrm{~GB}$ of RAM.

The cost introduced by sampling and evaluating the correlated transmittance with respect to classical transmittance is negligible in comparison to the cost of tracing samples. Simulation parameters and timings are shown in Table 1; note that negatively-correlated media tend to create longer paths, therefore increasing the total rendering cost. In terms of convergence, in some cases the pdf might not be proportional to the sampled transmittance [e.g. in Equation (25)], which in turn might increase variance; however we did not observe a strong effect in convergence when incorporating non-exponential transport. In Section S.10 in the supplemental we analyze the convergence experimentally.

Figure 1 shows volumetric renderings of translucent dragons made of materials with the same density, but different correlation. The middle image shows positive correlation, following a gamma distribution with $\operatorname{Var}(C)=40$. On the right we show negative correlation, exhibiting linear transmittance. In the three cases the media have scattering albedo $\Lambda=.8$, and mean extinction $\bar{\mu}=10 \mathrm{~m}^{-1}$. The net effect, due to the faster-than-exponential (negative correlation) and slower-than-exponential transmittance (positive correlation), is clearly visible in the final images.

Figure 13 highlights the versatility of our framework, with different scatterers correlation: negative correlation with linear transmittance decay, positive correlation according to our model, and an empirical distribution of $p(t)$ (modeled as a gamma distribution, see Section S.8 in the supplemental). The mean extinction is in all cases $\bar{\mu}=2 \mathrm{~m}^{-1}$, with albedo $\Lambda=.8$. Both the particles concentration and the cross section are isotropic. Figure 14 shows another non-exponential probability of extinction on granular compacted snow, using our model in Section 5.2. Optical parameters of the snow have been computed after Frisvad et al. [2007]. 


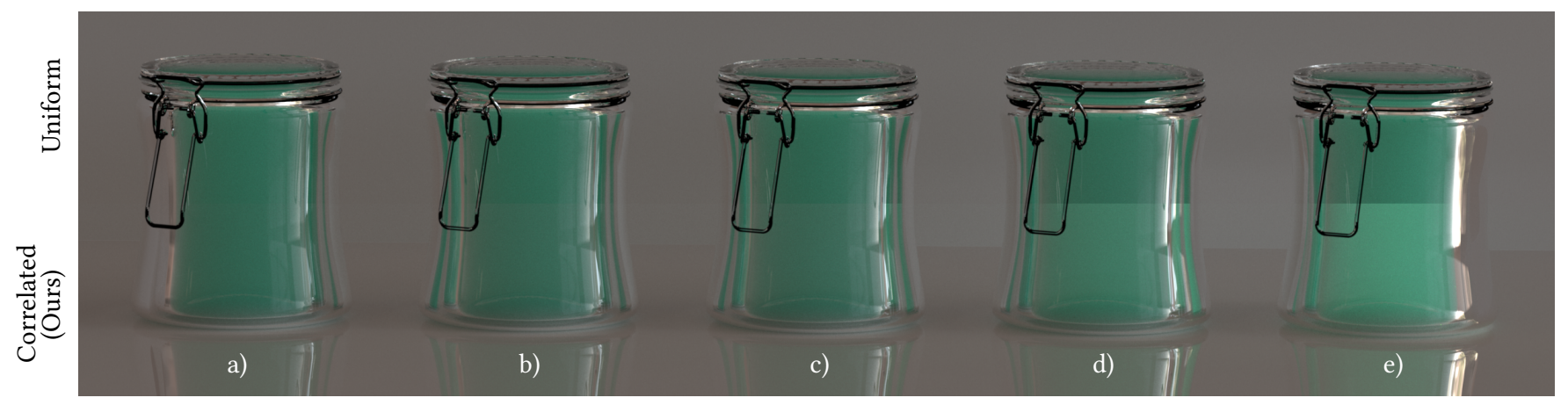

Fig. 15. Effect of local correlation in media with mean extinction $\bar{\mu}=2$ and albedo $\Lambda=\{.1, .8, .6\}$, and increasing $\operatorname{variance} \operatorname{Var}(C(\mathrm{x}))$ : a) 0 , b) 8 , c) 12 , d) 16 , and e) 32. The top half shows the result of classic light transport, while the bottom half shows the result of our model. $\operatorname{For} \operatorname{Var}(C(\mathbf{x}))=0$ the result is identical to classic light transport.
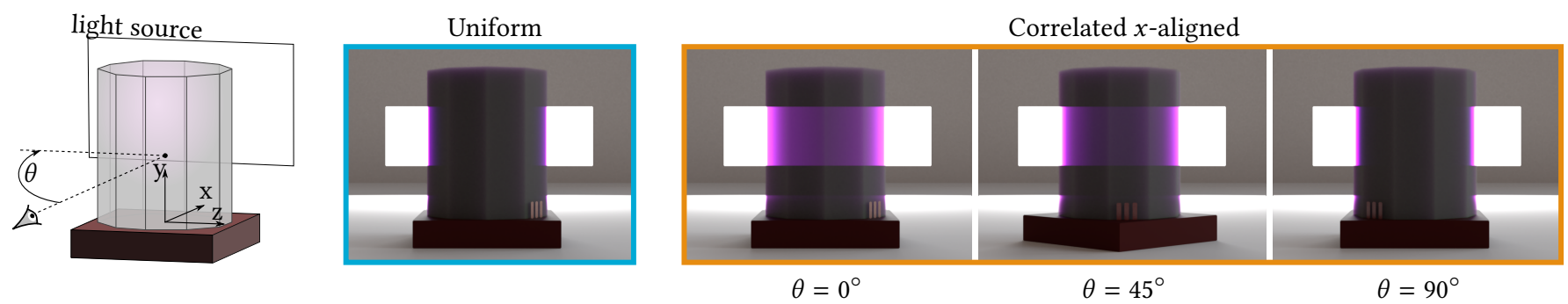

Fig. 16. Effect of directionally-dependent correlation on transmittance. The prism rotates around the $y$-axis. With uncorrelated media, appearance does not change with rotation. With a highly-correlated medium, appearance changes significantly as the prism rotates, according to the degree of alignment between the correlation and the view vector. The figure shows the case of $x$-axis-aligned correlation. Illustrative examples of the distribution of particles for each case are shown in Figure 4. Please refer to the supplemental for the video.

In Figure 15 we analyze the effect of correlation with increasing variance [increasing $\operatorname{Var}(C)$ in Equation (19)]. The top half of the jars has been rendered with the classic RTE, and thus remain constant independent of the degree of correlation, as expected. The bottom half shows the result of our model; note that for $\operatorname{Var}(C)=0$ the result converges to classic light transport.

Figure 16 shows the effect of directional correlation. The scene is made up of a volumetric prism with very low scattering albedo, so the dominant effect is transmittance, and a strong rectangular area light placed behind it. The prism rotates around its $y$-axis. The first prism is made up of an uncorrelated medium, while the other three show a strong positive correlation along the $x$-axis, with $\operatorname{Var}(C)=.5$; when the rotation angle is $\theta=0^{\circ}$, correlation is perfectly aligned with the $\mathrm{x}$-axis [similar to the situation depicted in Figure 4 (c)]. Both uncorrelated and correlated media have a mean particles concentration $\bar{C}=\{.8,1.6, .7\}$ (RGB), and a mean cross section $\bar{\sigma}=1$. For the uncorrelated medium, no changes occur in appearance as the prism rotates, as expected. For the $x$ aligned correlation, transmittance varies significantly as correlation progressively becomes unaligned with the view vector. We refer the reader to the supplemental video for the full animation, including other directions of correlation. Figure 17 systematically analyzes the effect of directional correlation for varying scattering albedo $\Lambda$, including uncorrelated media, isotropic correlated media, and directionally-correlated media aligned with the $x$ and $z$ axes ( $y$ being the up-vector). These four scenarios roughly correspond to the ones depicted in Figure 4.

Last, in Figure 18 we investigate if adjusting the optical parameters of an uncorrelated medium and using the classic RTE could produce the same results as our model for correlated media. In particular, we render the first statue with a correlated material (mean extinction $\bar{C}=20$, isotropic phase function, and scattering albedo $\Lambda=.8, .1, .1$ ), and render an uncorrelated version adjusting $\bar{C}=45$, with the same phase function and scattering albedo. Although tweaking the parameters of the RTE can lead to an overall similar appearance, it cannot correctly reproduce the details due to the different extinction curves and diffusive behavior in both models (see also [d'Eon 2014b]).

\section{CONCLUSIONS}

We have introduced a novel framework to simulate light transport in spatially-correlated media, where the probability of extinction and transmittance no longer follow an exponential decay, as predicted by the Beer-Lambert law. We have presented the Extended Generalized Boltzmann Equation, lifting the main limiting assumptions of the original GBE, and making it suitable for rendering applications. Our framework supports multiple sources, mixtures of particles, and directional correlation. In addition, we have proposed an intuitive model based on local optical properties for the most common case of positive correlation, providing a closed-form solution for 


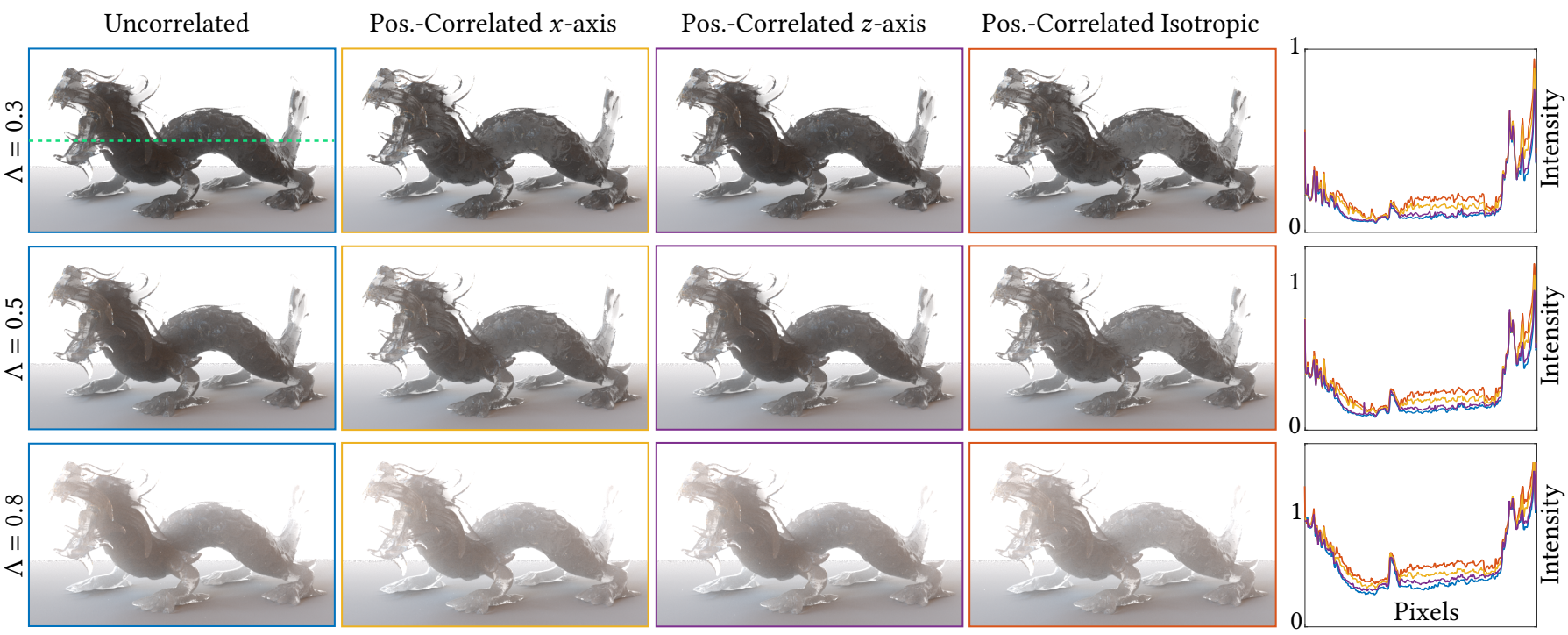

Fig. 17. Effect of directional correlation with varying scattering albedo $\Lambda$. From left to right: Uniformly distributed media, directionally-correlated in the $x$-axis (aligned to the camera view vector), directionally-correlated in $z$-axis, and isotropic correlation. For all cases we use positive correlation, and keep $\bar{C}=10$, $\sigma=1$, and $\operatorname{Var}(C)=10$ in the main axis of correlation, while for the remaining directions $\operatorname{Var}(C) \approx 0$ (so the mean free path is similar to the predicted by the Beer-Lambert law). To the right we plot intensity values (green scanline shown in one of the dragons) for each medium.

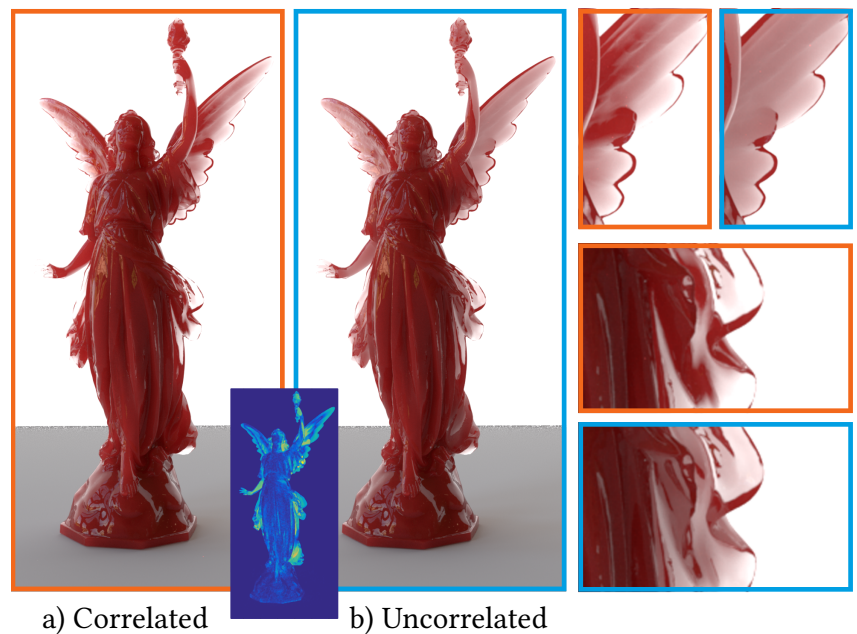

Fig. 18. Left: Render using our framework for correlated materials. Right: Render using the RTE, where the optical parameters of the material have been adjusted trying to match the appearance of the correlated case. Dissimilarities are evident, specially in thinner areas, since the extinction curves and diffusive behavior in both models are different (see false-color inset and zoomed-in areas).

transmittance, without the need for costly numerical simulation or precomputations, allowing to model $\Sigma(t)$ based on local definitions of $\mu$. Interestingly, Davis and Xu [2014] empirically proposed a similar expression to this model for transmittance in clouds. However, the authors stated that an integro-differential counterpart of their formulation was yet unknown. Our Equation (17) links this form of transmittance with the GBE, which is in turn an integro-differential equation.
Limitations and future work. Our theoretical framework in Section 4 is general, and supports heterogeneous media through the medium-to-medium boundary condition. However, practical implementation of such heterogeneities for continuous media is still challenging. This is because the differential probability of extinction $\Sigma(\mathbf{x})$ at point $\mathbf{x}$ affects $\Sigma\left(\mathbf{x}+\omega_{o} \mathrm{~d} t\right)$, according to the scatterers correlation at points $\mathbf{x}$ and $\mathbf{x}+\omega_{o} \mathrm{~d} t$, and to the cross-correlation between these two points. This means that the probability of extinction in correlated heterogeneous media cannot be modeled as the integral of the local differential extinction probabilities along the ray, as in uncorrelated heterogeneous media. In concurrent work, Camminady et al. [2017] proposed a solution for the simplest case, where the two media have identical structure $\eta_{1}=\eta_{2}=\eta_{1,2}$, and therefore the probability of extinction $p(t)$ only varies due to the different media density; however, finding an efficient, general solution remains a challenging problem. Revisiting numerical techniques for computing unbiased transmittance in heterogeneous media is thus an interesting topic of future work, since it is unclear how the underlying theory of virtual particles in existing methods [Coleman 1968; Kutz et al. 2017; Novák et al. 2014; Szirmay-Kalos et al. 2017; Woodcock et al. 1965] could be adapted to correlated scatterers. From a physical point of view, it would also be interesting to introduce in our Extended GBE [Equation (8)] support for refractive media [Ament et al. 2014; Gutierrez et al. 2006], as well as vector or bispectral scattering [Jarabo and Arellano 2018].

Other open problems include extending our local model [Equation (16)] to the case of negatively-correlated media, thus removing precomputation or the definition of an empirical $p(t)$, or finding a model for the continuous transition between correlated and particulate media. While for perfect negative correlation we can model light-particle interactions as a Bernoulli process (see Section S.8.1 
in the supplemental), for other degrees of negative correlation this process is not obvious: We hypothesize that such cases could be modeled as a mixture of Poissonian and Bernoulli processes, although an analytical model for negative correlation remains an open challenge that deserves a more in-depth exploration. Finally, for our final model we have chosen a gamma distribution for $p_{\tau}(\mu)$; however other probability distributions might work better depending on the scenario. Moreover, our directionally-resolved model for the variance of the distribution might be too smooth for materials with high-frequency details: in such cases, a mixture of ellipsoids (similar to the approach of Zhao et al. [2016]) could result in a more accurate fit.

Our definition of locally-correlated light transport may be suitable for filtering volumetric appearances, avoiding costly optimization procedures [Zhao et al. 2016], or ad-hoc shadowing functions [Schröder et al. 2011]. Accelerating rendering of particulate media [Meng et al. 2015; Müller et al. 2016] is another area that could benefit from our locally-correlated model. Introducing our compact representation into the shell transport functions proposed by Moon et al. [2007] and Müller et al. [2016] could significantly decrease the storage cost of these representations. Last, similarity theory [Wyman et al. 1989; Zhao et al. 2014] is an important tool for accelerating light transport within the RTE. Redefining this theory within locally-correlated radiative transport is another interesting avenue of work, specially given the additional degrees of freedom introduced by the non-exponential probability of extinction $p(t)$.

\section{A MIXTURES OF SCATTERERS}

For media made up of a mixture of scatterers $\mathcal{P}$, we compute the differential extinction probabilities $\Sigma(\mathbf{x}, t)$ (for both scatterer-toscatterer and source-to-scatterer transport) as

$$
\Sigma(\mathbf{x}, t)=\sum_{k \in \mathcal{P}} w_{k} \Sigma_{k}(\mathbf{x}, t),
$$

where the weights $w_{k}$ represent the probability of having a scatterer of type $k \in \mathcal{P}\left(\sum_{k \in \mathcal{P}} w_{k}=1\right)$, and $\Sigma_{k}(\mathbf{x}, t)$ is the differential extinction probability of each type. For the phase function and scattering albedo, we have

$$
\begin{gathered}
\Lambda(\mathbf{x}, t)=\sum_{k \in \mathcal{P}} \frac{w_{k} \Sigma_{k}(\mathbf{x}, t)}{\sum_{k \in \mathcal{P}} w_{k} \Sigma_{k}(\mathbf{x}, t)} \Lambda_{k}(\mathbf{x}, t), \\
f_{r}\left(\mathbf{x}, \omega_{i}, \omega_{o}, t\right)=\sum_{k \in \mathcal{P}} \frac{w_{k} \Sigma_{k}(\mathbf{x}, t) \Lambda_{k}(\mathbf{x}, t)}{\sum_{k \in \mathcal{P}} w_{k} \Sigma_{k}(\mathbf{x}, t) \Lambda_{k}(\mathbf{x}, t)} f_{r, k}\left(\mathbf{x}, \omega_{i}, \omega_{o}, t\right) .
\end{gathered}
$$

Last, using Equations (26) to (28), we can compute the scattering operator for a mixture of scatterers as

$$
\mathrm{B}\left(\mathbf{x}, \omega_{i}, \omega_{o}, t\right)=\sum_{k \in \mathcal{P}} w_{k} \mathrm{~B}_{k}\left(\mathbf{x}, \omega_{i}, \omega_{o}, t\right)
$$

\section{B MODELING DIRECTIONAL CORRELATION}

Similar to the anisotropy on the cross section described by Jakob et al. [2010], the scatterers correlation might also have an important directional effect, as illustrated in Figure 4 and observed by Vasques and Larsen [2014]. By considering the directional dependency on both $p_{C}(C)$ and $\sigma$, we transform Equation (18) into:

$$
\mu p_{\tau}\left(\mu ; \omega_{o}\right)=C p_{C}\left(C ; \omega_{o}\right) \sigma\left(\omega_{o}\right),
$$

where $p_{C}\left(C ; \omega_{o}\right)$ and $\bar{\sigma}$ are the probability distribution of the concentration and the mean cross section along $\omega_{o}$ respectively.

To model $p_{C}\left(C ; \omega_{o}\right)$, we noted that its only varying parameter is its variance, which we redefine as a directional function $\operatorname{Var}\left(C ; \omega_{o}\right) \in \Omega$. Following the same approach as the SGGX model [Heitz et al. 2015], we model $\operatorname{Var}\left(C ; \omega_{o}\right)$ as a zero-mean ellipsoid, using the matrix $\mathbf{V}$ defining the eigenspace of the variance of the projected concentration in $\Omega$ (see [Heitz et al. 2015] for details). We thus obtain:

$$
\operatorname{Var}\left(C ; \omega_{o}\right)=\sqrt{\omega_{o}^{T} \mathbf{V} \omega_{o}}
$$

For each direction $\omega_{o}$, we first obtain the projected variance, and then define the corresponding gamma distribution $\Gamma\left(C ; \alpha\left(\omega_{o}\right), \beta\left(\omega_{o}\right)\right)$ with $\alpha\left(\omega_{o}\right)$ and $\beta\left(\omega_{o}\right)$ computed from $\bar{C}$ and $\operatorname{Var}\left(C ; \omega_{o}\right)$. This has several benefits over other directional distributions: it is compact and efficient to evaluate; it supports anisotropy on the main axes; it is symmetric, smooth and non-negative in the full domain $\Omega$; and it is intuitive to characterize.

\section{ACKNOWLEDGMENTS}

We thank Miguel Angel Otaduy, Carlos Castillo and Jorge LopezMoreno for comments and discussions on early stages of the project and the dataset in Figure 11; Julio Marco, Adolfo Muñoz, and Ibón Guillén for discussions throughout the project; Pilar Romeo for help with the figures; all the members of the Graphics \& Imaging Lab that helped with proof-reading; and the reviewers for the in-depth reviews. The ICEBERG was modeled by Tora2097 from TurboSquid, while the DrAgON and Lucy are from the Stanford 3D Scanning Repository. This project has been funded by the European Research Council (ERC) under the EU's Horizon 2020 research and innovation programme (project CHAMELEON, grant No 682080), DARPA (project REVEAL), and the Spanish Ministerio de Economía y Competitividad (projects TIN2016-78753-P and TIN2014-61696-EXP).

\section{REFERENCES}

Carlos Aliaga, Carlos Castillo, Diego Gutierrez, Miguel A. Otaduy, Jorge Lopez-Moreno, and Adrian Jarabo. 2017. An Appearance Model for Textile Fibers. Computer Graphics Forum (Proc. EGSR 2017) 36, 4 (2017).

John Amanatides and Andrew Woo. 1987. A fast voxel traversal algorithm for ray tracing. In Eurographics, Vol. 87. 3-10.

Marco Ament, Christoph Bergmann, and Daniel Weiskopf. 2014. Refractive radiative transfer equation. ACM Trans. Graph. 33, 2 (2014)

James Arvo. 1993. Transfer equations in global illumination. SIGGRAPH '93 Course Notes 2 (1993).

Howard W Barker, Bruce A Wiellicki, and Lindsay Parker. 1996. A parameterization for computing grid-averaged solar fluxes for inhomogeneous marine boundary layer clouds. Part II: Validation using satellite data. Journal of the Atmospheric Sciences 53, 16 (1996).

Fabien Bellet, Elie Chalopin, Florian Fichot, Estelle Iacona, and Jean Taine. 2009. RDFI determination of anisotropic and scattering dependent radiative conductivity tensors in porous media: Application to rod bundles. International fournal of Heat and Mass Transfer 52, 5 (2009), 1544-1551.

Thomas Camminady, Martin Frank, and Edward W. Larsen. 2017. Nonclassical Particle Transport in Heterogeneous Materials. In International Conference on Mathematics \& Computational Methods Applied to Nuclear Science \& Engineering.

Subrahmanyan Chandrasekhar. 1960. Radiative Transfer. Dover.

WA Coleman. 1968. Mathematical verification of a certain Monte Carlo sampling technique and applications of the technique to radiation transport problems. Nuclear science and engineering 32, 1 (1968), 76-81. 
R Coquard and D Baillis. 2006. Radiative properties of dense fibrous medium containing fibers in the geometric limit. Journal of heat transfer 128, 10 (2006), 1022-1030.

Anthony B Davis and Alexander Marshak. 2004. Photon propagation in heterogeneous optical media with spatial correlations: enhanced mean-free-paths and widerthan-exponential free-path distributions. Fournal of Quantitative Spectroscopy and Radiative Transfer 84, 1 (2004).

Anthony B Davis, Alexander Marshak, H Gerber, and Warren J Wiscombe. 1999. Horizontal structure of marine boundary layer clouds from centimeter to kilometer scales. Fournal of Geophysical Research: Atmospheres 104, D6 (1999).

Anthony B Davis and Mark B Mineev-Weinstein. 2011. Radiation propagation in random media: From positive to negative correlations in high-frequency fluctuations. Fournal of Quantitative Spectroscopy and Radiative Transfer 112, 4 (2011).

Anthony B Davis and Feng Xu. 2014. A Generalized Linear Transport Model for Spatially Correlated Stochastic Media. Journal of Computational and Theoretical Transport 43, 1-7 (2014).

Eugene d'Eon. 2014a. Computer graphics and particle transport: our common heritage, recent cross-field parallels and the future of our rendering equation. In Digipro 2014

Eugene d'Eon. 2014b. Rigorous asymptotic and moment-preserving diffusion approximations for generalized linear Boltzmann transport in arbitrary dimension. Transport Theory and Statistical Physics 42, 6-7 (2014), 237-297.

Eugene d'Eon. 2016a. Diffusion approximations for nonclassical Boltzmann transport in arbitrary dimension. Technical Report.

Eugene d'Eon. 2016b. A Hitchhiker's Guide to Multiple Scattering.

Martin Frank and Thierry Goudon. 2010. On a generalized Boltzmann equation for non-classical particle transport. Kinetic and Related Models 3 (2010).

Jeppe Revall Frisvad, Niels Jørgen Christensen, and Henrik Wann Jensen. 2007. Computing the scattering properties of participating media using Lorenz-Mie theory. ACM Trans. Graph. 26, 3 (2007)

Giovanni Gallavotti. 1972. Rigorous Theory Of The Boltzmann Equation In The Lorentz Gas. Technical Report. Istituto di Fisica, Univ. di Roma.

Diego Gutierrez, Adolfo Munoz, Oscar Anson, and Francisco Seron. 2006. Simulation of Atmospheric Phenomena. Computers \& Graphics 20, 6 (2006), 994:1010.

Diego Gutierrez, Srinivasa G. Narasimhan, Henrik Wann Jensen, and Wojciech Jarosz. 2008. Scattering. In ACM SIGGRAPH ASIA 2008 Courses.

Eric Heitz, Jonathan Dupuy, Cyril Crassin, and Carsten Dachsbacher. 2015. The SGGX Microflake Distribution. ACM Trans. Graph. 34, 4, Article 48 (2015). http://doi.acm. org $/ 10.1145 / 2766988$

Wenzel Jakob. 2010. Mitsuba renderer. http://www.mitsuba-renderer.org.

Wenzel Jakob, Adam Arbree, Jonathan T Moon, Kavita Bala, and Steve Marschner. 2010. A radiative transfer framework for rendering materials with anisotropic structure. ACM Trans. Graph. 29, 4 (2010)

Adrian Jarabo and Victor Arellano. 2018. Bidirectional Rendering of Vector Light Transport. Computer Graphics Forum To appear (2018).

Wojciech Jarosz, Derek Nowrouzezahrai, Iman Sadeghi, and Henrik Wann Jensen. 2011 A Comprehensive Theory of Volumetric Radiance Estimation Using Photon Points and Beams. ACM Trans. Graph. 30, 1 (2011).

Henrik Wann Jensen. 2001. Realistic Image Synthesis Using Photon Mapping. AK Peters.

Pramook Khungurn, Daniel Schroeder, Shuang Zhao, Kavita Bala, and Steve Marschner 2015. Matching Real Fabrics with Micro-Appearance Models. ACM Trans. Graph 35, 1 (2015).

Yuri Knyazikhin, JöRn Kranigk, Ranga B Myneni, Oleg Panfyorov, and Gode Gravenhorst. 1998. Influence of small-scale structure on radiative transfer and photosynthesis in vegetation canopies. Fournal of Geophysical Research 103 (1998), 6133-6144.

Alexander B Kostinski. 2001. On the extinction of radiation by a homogeneous but spatially correlated random medium. FOSA A 18, 8 (2001).

Alexander B Kostinski. 2002. On the extinction of radiation by a homogeneous but spatially correlated random medium: reply to comment. FOSA A 19, 12 (2002), 2521-2525.

Jaroslav Křivánek, Iliyan Georgiev, Toshiya Hachisuka, Petr Vévoda, Martin Šik, Derek Nowrouzezahrai, and Wojciech Jarosz. 2014. Unifying points, beams, and paths in volumetric light transport simulation. ACM Trans. Graph. 33, 4 (2014).

Peter Kutz, Ralf Habel, Yining Karl Li, and Jan Novák. 2017. Spectral and Decomposition Tracking for Rendering Heterogeneous Volumes. ACM Trans. Graph. 36, 4 (2017).

Eric P Lafortune and Yves D Willems. 1996. Rendering participating media with bidirectional path tracing. In Rendering Techniquesâ̆ $\breve{Z} 96$.

Edward W Larsen. 2007. A generalized Boltzmann equation for non-classical particle transport. In Proceedings of the International Conference on Mathematics and Computations and Supercomputing in Nuclear Applications.

Edward W Larsen and Richard Vasques. 2011. A generalized linear Boltzmann equation for non-classical particle transport. Fournal of Quantitative Spectroscopy and Radiative Transfer 112, 4 (2011)

C D Levermore, G C Pomraning, D L Sanzo, and J Wong. 1986. Linear transport theory in a random medium. Fournal of mathematical physics 27, 10 (1986).

Jorge Lopez-Moreno, David Miraut, Gabriel Cirio, and Miguel A. Otaduy. 2015. Sparse GPU Voxelization of Yarn-Level Cloth. Computer Graphics Forum 36, 1 (2015).
Guillaume Loubet and Fabrice Neyret. 2017. Hybrid mesh-volume LoDs for all-scale pre-filtering of complex 3D assets. Computer Graphics Forum 36 (2017).

S Lovejoy, G Brosamlen, and B Watson. 1995. Scattering in multifractal media. In Particle Transport in Stochastic Media.

Alexander Marshak, Anthony Davis, Warren Wiscombe, and Robert Cahalan. 1998. Radiative effects of sub-mean free path liquid water variability observed in stratiform clouds. Journal of Geophysical Research: Atmospheres 103, D16 (1998), 19557-19567.

Johannes Meng, Marios Papas, Ralf Habel, Carsten Dachsbacher, Steve Marschner, Markus Gross, and Wojciech Jarosz. 2015. Multi-Scale Modeling and Rendering of Granular Materials. ACM Trans. Graph. 34, 4 (2015).

Jonathan T Moon, Bruce Walter, and Stephen R Marschner. 2007. Rendering discrete random media using precomputed scattering solutions. In Proceedings of EGSR.

Thomas Müller, Marios Papas, Markus Gross, Wojciech Jarosz, and Jan Novák. 2016. Efficient Rendering of Heterogeneous Polydisperse Granular Media. ACM Trans. Graph. 35, 6 (2016)

William I Newman, Jeffrey K Lew, George L Siscoe, and Robert G Fovell. 1995. Systematic effects of randomness in radiative transfer. Journal of the atmospheric sciences 52, 4 (1995).

Fabrice Neyret. 1998. Modeling, animating, and rendering complex scenes using volumetric textures. IEEE Transactions on Visualization and Computer Graphics 4, 1 (1998), 55-70.

Jan Novák, Andrew Selle, and Wojciech Jarosz. 2014. Residual Ratio Tracking for Estimating Attenuation in Participating Media. ACM Trans. Graph. 33, 6 (2014).

Jouni I Peltoniemi and Kari Lumme. 1992. Light scattering by closely packed particulate media. 7OSA A 9, 8 (1992).

Iman Sadeghi, Adolfo Munoz, Philip Laven, Wojciech Jarosz, Francisco Seron, Diego Gutierrez, and Henrik Wann Jensen. 2012. Physically-based simulation of rainbows. ACM Trans. Graph. 31, 1 (2012).

Kai Schröder, Reinhard Klein, and Arno Zinke. 2011. A Volumetric Approach to Predictive Rendering of Fabrics. Computer Graphics Forum 30, 4 (2011).

László Szirmay-Kalos, Iliyan Georgiev, Milán Magdics, Balázs Molnár, and Dávid Légrády. 2017. Unbiased Estimators to Render Procedurally Generated Inhomogeneous Participating Media. Computer Graphics Forum 36, 2 (2017). EUROGRAPHICS 2017.

Jean Taine, Fabien Bellet, Vincent Leroy, and Estelle Iacona. 2010. Generalized radiative transfer equation for porous medium upscaling: Application to the radiative Fourier law. International fournal of Heat and Mass Transfer 53, 19 (2010), 4071-4081.

Richard Vasques and Edward W Larsen. 2014. Non-classical particle transport with angular-dependent path-length distributions. I: Theory. Annals of Nuclear Energy 70 (2014), 292-300.

Eric Veach. 1997. Robust Monte Carlo methods for light transport simulation. Ph.D. Dissertation. Stanford.

E. Woodcock, T. Murphi, P. Hemmings, and S. Longworth. 1965. Techniques used in the GEM code for Monte Carlo neutronics calculations in reactors and other systems of complex geometry.. In Proc. Conf. Applications of Computing Methods to Reactors, ANL-7050.

Magnus Wrenninge, Ryusuke Villemin, and Christophe Hery. 2017. Path Traced Subsurface Scattering using Anisotropic Phase Functions and Non-Exponential Free Flights. Technical Report Pixar Technical Memo 17-07. Pixar Inc.

Douglas R Wyman, Michael S Patterson, and Brian C Wilson. 1989. Similarity relations for the interaction parameters in radiation transport. Applied optics 28, 24 (1989), 5243-5249.

Shuang Zhao, Wenzel Jakob, Steve Marschner, and Kavita Bala. 2011. Building volumetric appearance models of fabric using micro CT imaging. ACM Trans. Graph. 30 4 (2011).

Shuang Zhao, Wenzel Jakob, Steve Marschner, and Kavita Bala. 2012. Structure-aware synthesis for predictive woven fabric appearance. ACM Trans. Graph. 31, 4 (2012).

Shuang Zhao, Ravi Ramamoorthi, and Kavita Bala. 2014. High-order similarity relations in radiative transfer. ACM Transactions on Graphics (TOG) 33, 4 (2014).

Shaung Zhao, Lifan Wu, Frédo Durand, and Ravi Ramamoorthi. 2016. Downsampling Scattering Parameters for Rendering Anisotropic Media. ACM Trans. Graph. 35, 6 (2016) 